\title{
Major Axis Cross-sectional Diameter at End Ventricular Diastole
}

National Cancer Institute

\section{Source}

National Cancer Institute. Major Axis Cross-sectional Diameter at End Ventricular

Diastole. NCI Thesaurus. Code C127573.

The cross sectional diameter of a cardiovascular structure measured along the major axis at end ventricular diastole. 